\title{
Transatlantica
}

Revue d'études américaines. American Studies Journal

\section{Sarah Hatchuel, Lost, fiction vitale, Paris, PUF, 2012, 145 pages}

\section{Bernard Genton}

\section{(2) OpenEdition}

1 Journals

\section{Édition électronique}

URL : https://journals.openedition.org/transatlantica/6056

DOI : 10.4000/transatlantica.6056

ISSN : 1765-2766

Éditeur

Association française d'Etudes Américaines (AFEA)

\section{Référence électronique}

Bernard Genton, «Sarah Hatchuel, Lost, fiction vitale, Paris, PUF, 2012, 145 pages », Transatlantica [En ligne], 2 | 2012, mis en ligne le 25 mai 2013, consulté le 06 avril 2023. URL : http:// journals.openedition.org/transatlantica/6056 ; DOI : https://doi.org/10.4000/transatlantica.6056

Ce document a été généré automatiquement le 6 avril 2023.

\section{(c)}

Creative Commons - Attribution - Pas d'Utilisation Commerciale - Pas de Modification 4.0 International - CC BY-NC-ND 4.0

https://creativecommons.org/licenses/by-nc-nd/4.0/ 


\section{Sarah Hatchuel, Lost, fiction vitale, Paris, PUF, 2012, 145 pages}

\section{Bernard Genton}

L'industrie télévisuelle américaine produit depuis quelques années des séries qui rencontrent un succès public et critique grandissant et qui constituent souvent la culture commune des étudiants d'aujourd'hui. Il est donc tout à fait normal que l'université se penche à son tour sur ce phénomène culturel. En France, un certain nombre de pionniers, à l'Université du Havre, à Rouen, à Paris 7 et dans d'autres centres de recherche ont ouvert ce nouveau champ d'exploration. Sarah Hatchuel, spécialiste reconnue de Shakespeare au cinéma, est l'une des initiatrices de ce mouvement, et il est donc tout naturel que son ouvrage sur Lost paraisse dans la série (d'études universitaires) que viennent de lancer les PUF, avec pour objectif « d'analyser [ces] objets culturels, de comprendre les raisons de leur prospérité et d'en apporter les clés de lecture ».

Diffusée sur la chaîne commerciale ABC de 2004 à 2010 (et sur TF1 en France à partir de 2005), Lost se développa sur six saisons comprenant au total 121 épisodes de 42 minutes. La série rencontra rapidement un très grand succès aux États-Unis puis dans le monde, puisque 200 pays finirent par acheter les droits de diffusion. L'argument de base est simple, classique même, puisqu'il s'agit au départ de présenter la vie d'une quarantaine de passagers rescapés d'un accident d'avion : ils se retrouvent sur une île $\mathrm{du}$ Pacifique, apparemment déserte, mais en réalité habitée par des phénomènes étranges et des créatures terrifiantes, par exemple ces ours polaires égarés sous les tropiques que l'on voit à plusieurs reprises dans la première saison, mais aussi par d'autres humains, que le spectateur découvre peu à peu, et tout particulièrement par un groupe à l'origine indéterminée, les "Autres »: comme le note l'auteur dans la « fiche d'identité » liminaire, ces passagers devront «affronter les Autres, apprendre à vivre ensemble, se racheter, se transcender, mais aussi à décrypter (et finalement protéger) les mystères de l'île, et conjurer leur propre perte de repères et leur passé tourmenté pour mieux envisager l'avenir, voire un autre monde ». On remarque aussi, dès les premiers épisodes, la récurrence d'un certain nombre d'expressions ou 
d'aphorismes qui voyagent tout au long de la série, passant parfois d'un personnage à l'autre : « Don't tell me what I can't do ", "What happened happened », «We've got to get back ", « Do not mistake coincidence for fate ", « Live together, die alone », « We're here $» . .$.

Pour Sarah Hatchuel, Lost est une "série culte " qui se distingue par son interactivité impérative : «Lost a besoin de nous, écrit-elle, à la fois individuellement et collectivement, et a profondément transformé nos attentes en termes de complexité narrative dans les séries télévisées " (9). En effet, Lost s'est donné tous les ingrédients pour "perdre » le spectateur à force d' "expérimentations» et de "confusions narratives ». S'appuyant sur ces constatations initiales, Sarah Hatchuel procède méthodiquement, par approfondissements successifs, en trois chapitres, dont les titres reprennent trois des leitmotiv que nous venons d'évoquer. Le premier (« Nous devons y retourner ») met en perspective les traits les plus saillants du produit. Pour commencer, l'instabilité temporelle et géographique instituée par la prolifération des flash-backs puis, à partir de la fin de la troisième saison, des flash-forwards, installe une conscience permanente du passé dans le présent, bref cette nostalgie, ce besoin irrépressible d'un retour vers un "avant» idéalisé. Par ailleurs, un intertexte postmoderne de citations et d'allusions à la fois philosophiques, littéraires, télévisuelles et cinématographiques » (21) affirme le caractère hybride de la série, son épaisseur culturelle tout en laissant le spectateur libre de s'y retrouver, ou non... Comme d'autres séries, mais peut-être plus qu'elles, Lost connaît aussi une amplification médiatique qui l'accompagne tout au long de son existence, et qui lui survit après la diffusion de l'ultime épisode en 2010 : forums de discussion, sites dédiés créés par des fans, mais aussi tactiques de commercialisation, DVD, streaming, courtes séquences accessibles sur les téléphones portables («mobisodes»), tout ceci contribuant, selon Sarah Hatchuel, à la création de cette " hypersérialité où la fiction s'immisce et s'expérimente dans le réel» (24-5). Enfin, et c'est selon l'auteur un élément central, la série est parcourue de bout en bout par une "esthétique de la surprise", qui commence par l'utilisation systématique et méthodique du procédé du cliffhanger, élément narratif qui vient interrompre le cours du récit pour tout remettre en question, de préférence avant la pause publicitaire, c'est-à-dire «toutes les douze minutes environ » (34). Ici, l'effet recherché va bien au-delà de l'objectif commercial, puisqu'il s'agit toujours de préserver la liberté du spectateur: à lui d'établir les rapports, de trouver les explications, de se fabriquer sa propre « théorie narrative » (45).

Dans le second chapitre («Ce qui est arrivé est arrivé»), Sarah Hatchuel poursuit son chemin en se penchant sur les rapports complexes institués par la série Lost entre la narration (ou l'écriture) et l'expérience individuelle, celle des personnages, mais aussi celle des spectateurs qui se trouvent ainsi mis à contribution, puisque les expériences des personnages renvoient aussi aux nôtres, la série mettant en scène de manière systématique "cette tension entre récit éminemment travaillé (qui se conjugue au passé et au futur) et impression de vivre le surgissement et le retournement en permanence (qui se conjugue au présent)» (55). On pourrait aussi penser que Lost joue sur l'un des ressorts les plus éprouvés de la narration fantastique, à savoir la possibilité des voyages dans le temps. Le passé peut-il être changé ? La série affirme que non, tout en jouant résolument sur ce fantasme, ce que vient confirmer la sixième saison, entièrement construite sur des hypothèses narratives radicalement différentes, puisque le spectateur y voit les personnages principaux emprunter d'autres 
voies, choisir d'autres options et même changer de vie(s) - comme si le choix existait : si le spectateur est d'abord désarçonné par les flash-sideways, il s'y habitue rapidement.

Les aperçus anthropologiques et politiques qui constituent pour l'essentiel le troisième et dernier chapitre du livre («Vivre ensemble ou mourir seul ») enrichissent l'analyse et élargissent la perspective. Pour Sarah Hatchuel, la série a en effet des choses à dire sur les rapports entre les genres et d'une manière plus générale sur le vivre ensemble, comme toutes les fictions iliennes qui l'ont précédée. Dans le cas de Lost, nous explique Sarah Hatchuel, nous sommes en présence d'un point de vue résolument masculin : c'est un "récit classique de masculinité blessée mais résiliente ", animée de surcroît d'un patriotisme étatsunien apaisé, puisque le modèle américain ("le droit, la loi, la science, la raison») se trouve mis en perspective et modifié par l'expérience que traversent les rescapés sur l'île, où s'élabore un modèle « communautaire, multiracial et post-national » qui n'est pas antithétique : comme le note Sarah Hatchuel, c'est « au bout du compte un récit d'assimilation des cultures par la nation américaine, dans lequel le mythe du melting pot et d'un société soi-disant tolérante et non impérialiste resurgit avec vigueur » (100-1). Et c'est sans doute dans le traitement finalement nuancé de la notion d'altérité que la série affirme son parti-pris idéologique positif : si les Autres sont en règle générale privés de flashbacks individuels, deux ou trois d'entre eux font malgré tout l'objet d'un examen plus attentif, qui permet au spectateur de mieux «comprendre leur comportement présent» et leur manichéisme conformiste, même si la série a besoin en permanence de générer de «nouveaux ennemis ».

Dans une conclusion d'un ton moins retenu que le reste de la démonstration, l'auteur affirme, avec d'autres, que «l'expérience de Lost devient une expérience quasi existentielle, englobant ses créateurs, ses acteurs, ses spectateurs » (132), enthousiasme que confirme la dernière phrase de l'ouvrage : «Si les spectateurs ont donné beaucoup de leur temps, de leur vie, de leur énergie (à comprendre, chercher, décoder) la série leur a rendu leur propre vie (peut-être changée, voire transcendée) en retour » (134).

Un esprit chagrin, ou rationaliste, n'est peut-être pas obligé d'aller jusque-là. Mais l'on sait également gré à Sarah Hatchuel d'épargner au lecteur les considérations ésotériques, gnostiques ou métaphysiques, et de restreindre ses observations et ses analyses à des éléments plus tangibles. Tout au plus pourrait-on lui reprocher une trop grande indulgence pour une œuvre certes foisonnante et pleine d'inventivité. Faut-il pour autant exclure l'hypothèse que le «chaos narratif et idéologique » de la série (133), pour intéressant qu'il puisse paraître, n'est rien d'autre que cela, c'est-à-dire le résultat d'une improvisation permanente, souvent d'une très grande ingéniosité, mais où les ficelles restent souvent visibles, et dont les fins ultimes restent commerciales ? Observons qu'il serait également intéressant de se pencher sur la mentalité des fabricateurs et sur les processus d'écriture et de production de la trame narrative : les producteurs eux-mêmes donnent de nombreux indices, parfois sur le mode ironique, par exemple lorsqu'ils n'hésitent pas à enterrer vivants deux personnages dont le profilage était sans issue et la réception par le public négative - il s'agit des amants diaboliques Nikki et Paolo qui surgissent puis disparaissent rapidement dans la troisième saison.

Læ conception initiale de Lost serait due à Lloyd Brown, patron de la chaîne $\mathrm{ABC}$, à un moment où celui-ci effectuait un séjour à Hawaï au début des années $2000^{1}$ : ce haut dirigeant audiovisuel eut alors l'idée d'une série qui combinerait les 
expériences respectives du roman anglais Lord of the Flies (William Golding, 1957), des films qui en ont été tirés (Peter Brook, 1963 et Harry Hook, 1990), de la sitcom Gilligan's Island (diffusée sur CBS entre 1964 et 1967), du film Cast Away (Robert Zemeckis, 2000) et du programme de reality TV Survivor (concept britannique à l'origine, mis en œuvre en Suède puis aux États-Unis à la fin des années 1990). Peut-être pourrait-on regretter que Sarah Hatchuel n'accorde pas une attention plus soutenue à la matérialité de Lost. C'est une série de network, ce qui a des conséquences esthétiques non négligeables: un langage contrôlé, une certaine pruderie, une maîtrise rusée de la corporalité. Si l'on voit le sang dans Lost, et parfois des blessures impressionnantes - fractures ouvertes, ventres transpercés, on n'aperçoit aucun sein ni aucune fesse. Mais la pruderie postmoderne n'empêche pas une tension érotique permanente, dont les ingrédients sont souvent plus proches du style de la téléréalité que du cinéma de l'âge d'or: Tshirts mouillés, femmes en soutien-gorge, corps luisants, scènes de lit dans les flashbacks, etc.

Læ nécessité, fondamentale pour la télévision commerciale, de garantir un public aussi nombreux que possible aux annonceurs influe fortement sur la structure narrative des séries : c'est même la fonction principale de ces cliffhangers qui rythment les épisodes toutes les douze minutes. Cet impératif explique également le délayage fréquent de l'action, les répétitions ou résumés parfois laborieux, mais désormais canoniques ("Previously in Lost...»). Le plus gênant dans ce travail de série, c'est évidemment l'appareil idéologico-métaphysique. La mise en parallèle du réalisme (essentiellement les flashbacks) et de la magie et du fantastique (ce qui se passe sur l'île, les flash-forwards ou -sideways) confère à l'ensemble, par endroits, une atmosphère grand-guignolesque qui rend d'autant plus hasardeuses les interprétations philosophiques ou métaphysiques. La parole de John Locke s'adressant à Jack à la fin de la première saison: "You're a man of science, I'm a man of faith", enferme la série dans un dilemme simpliste, sans parler des tentations numérologiques, ésotériques et astrologiques auxquelles elle ne cesse de succomber, suscitant par-là même d'innombrables commentaires sur internet. De ce point de vue, le premier «film d'orientation " proposé dans la mise en abyme de la série (S3, E2), et projeté pour Locke et Jack, est assez révélateur : le " projet Dharma ", l'un des mystères à demi dévoilés de l'île sans nom, y est présenté comme un vaste programme commun de recherche universelle, mêlant « la météorologie, la psychologie, la parapsychologie, la zoologie et l'électromagnétisme ». On peut donner raison à Pacôme Thiellement, commentateur inspiré, pour ne pas dire exalté, de Lost, qui voit ici une mise en abyme de la série tout entière ${ }^{2}$. Mais faut-il pour autant prendre au sérieux cette " orientation »?

Dans ce livre court et stimulant, écrit dans une langue claire et alerte, Sarah Hatchuel propose une analyse serrée, à la fois sur le plan narratologique et sur le plan culturel, de l'une des séries les plus marquantes des années 2000. Elle permet au lecteur de mieux cerner les raisons de la séduction qu'a pu exercer Lost auprès d'un très large public mondialisé. Si l'on n'est pas obligé de partager en tous points l'admiration de l'auteur, on ne peut que remercier celle-ci pour cet essai passionnant à lire, et qui démontre avec brio et finesse que le nationalisme culturel américain brille encore de tous ses feux, même dans la version modérée et respectueuse des différences que l'on trouve ici. Le final est une scène de retrouvailles dans la lumière douce d'une église qui figure l'au-delà. 


\section{NOTES}

1. Cf. "Lost, How It All Began », Entertainment Weekly, 7 mai 2010, www.ew.com, et l'article Wikipedia version anglaise, consulté le 4 mars 2010.

2. Pacôme Thiellement, http://www.dailymotion.com/video/xqbagt_lost-la-serie-analysee-parpacome-thiellement_tv\#.UTc5ajcg91U, consulté le 4 mars 2013.

INDEX

Thèmes : Recensions

\section{AUTEUR}

\section{BERNARD GENTON}

Université de Strasbourg 